\title{
Certain generating matrix functions of Charlier matrix polynomials using Weisner's group theoretic method
}

\author{
Ayman Shehata * \\ Department of Mathematics, Faculty of Science, Assiut University, Assiut 71516, Egypt. \\ Department of Mathematics, College of Science and Arts, Unaizah, Qassim University, \\ Qassim, Kingdom of Saudi Arabia.
}

\begin{abstract}
The present paper discusses a study of a class of Charlier matrix polynomials and its generalized analogue. Certain generating matrix functions, recurrence matrix relations, matrix differential equation, summation formulas and many new results have been discussed for these matrix polynomials. Weisner's group theoretic method is used to obtain matrix generating relations for Charlier matrix polynomials and the details of this method were given in this paper. Finally, we will discuss only briefly the procedure followed.
\end{abstract}

AMS Mathematics Subject Classification(2010): 22E60; 33C45; 33C65; $15 \mathrm{~A} 60$.

Keywords and phrases: Charlier matrix polynomials; Generating matrix functions; Charlier matrix differential equations; Lie algebra.

\section{Introduction and motivation}

Carl Charlier [2,13] introduced the family of scalar Charlier polynomials. The introduction of special functions is used as an analytical foundation for the majority of problems in mathematical physics it has been solved exactly and finds broad practical applications. With the advancement of knowledge in the field of special functions, it has been the approach of past years of research work to search for new and easy approaches to establish new results and to give easy methods to obtain certain already unknown, known and new results. This approach is better known as the Lie theoretic method. The first significant advancement in this direction was made by Weisner $[21,22,23,24,25,26]$ who exhibits the group theoretic significance of generating functions for hypergeometric, Hermite, and Bessel functions. Then Manocha [8], Manoche and Jain [9], Miller [11, 12] and McBride [10] present Weisner's method in a systematic manner and thereby laid its firm foundation. Miller also extends Weisner's theory by relating it to what? Recently the matrix analogues of Laguerre and modified Laguerre matrix polynomials have been considered in $[5,6,7,16,17]$ for matrices in $\mathbb{C}^{N \times N}$ in a number of previous papers, see for example $[1,14,15]$ and his work references therein $[18,19,20]$.

Motivated by the important works mentioned above, in the present paper mainly the group theoretic approach of Weisner who has been used Weisner devised a method for obtaining generating matrix relations for Charlier matrix polynomials, which satisfy certain conditions. In Section 2, we give the definition, many new results and known properties of Charlier matrix polynomials. In Section 3, we use the representation theory of the Lie algebra method and derive certain generating matrix relations involving these matrix polynomials by using Weisner's group theoretic techniques.

\subsection{Preliminaries}

In this subsection, we review some useful definitions, lemmas and the concepts related to the matrix functional calculus. Throughout this paper, for a matrix $A$ in $\mathbb{C}^{N \times N}$, its spectrum is denoted by $\sigma(A)$ where $\sigma(A)$ is the set of the eigenvalues of $A$. The matrices $I$ and $\mathbf{0}$ will denote the identity matrix and null matrix in $\mathbb{C}^{N \times N}$, respectively.

\footnotetext{
${ }^{*}$ Corresponding author. E-mail: drshehata2006@yahoo.com
} 
Definition 1.1. [3, 4] For a matrix $A \in \mathbb{C}^{N \times N}$ such that $\sigma(A)$ does not contain 0 or a negative integer $\left(\sigma(A) \cap \mathbb{Z}^{-}=\emptyset\right.$ where $\emptyset$ is an empty set), the matrix form of the shifted factorial is defined by

$$
(A)_{n}= \begin{cases}A(A+I) \ldots(A+(n-1) I)=\Gamma(A+n I) \Gamma^{-1}(A), & n \geq 1 \\ I, & n=0\end{cases}
$$

where $\Gamma(A)$ is an invertible matrix in $\mathbb{C}^{N \times N}$ and $\Gamma^{-1}(A)$ is inverse Gamma matrix function.

For $A$ is an arbitrary matrix of $\mathbb{C}^{N \times N}$ and using (1.1), we have the relations

$$
\begin{aligned}
& (A)_{n+k}=(A)_{n}(A+n I)_{k}=(A)_{k}(A+k I)_{n}, \\
& (-n I)_{k}= \begin{cases}\frac{(-1)^{k} n !}{(n-k) !} I, & 0 \leq k \leq n \\
\mathbf{0}, & k>n .\end{cases}
\end{aligned}
$$

If $\operatorname{Re}(\mu) \in \sigma(A)$ is not an integer and using (1.1), we have the relation

$$
\Gamma(I-A-n I) \Gamma^{-1}(I-A)=(-1)^{n}\left[(A)_{n}\right]^{-1},
$$

where $\Gamma(I-A)$ is an invertible matrix. In view of Eq. (1.1) and (1.3), we observe that

$$
\Gamma(A+I) \Gamma^{-1}(A-(k-1) I)=(-1)^{k}(-A)_{k}
$$

where $\Gamma(A-(k-1) I)$ is an invertible matrix.

\section{Charlier matrix polynomials: Definition and properties}

Let us assume that $A$ is a matrix in $\mathbb{C}^{N \times N}$ whose $A$ satisfy the condition $-k \notin \sigma(A)$, for every integer $k>0(\sigma(A)=$ spectrum of $A), \lambda$ is a complex parameter whose real part is a positive and let us consider a new generating matrix function which represents the Charlier matrix polynomials $\mathbf{C}_{n}(A ; \lambda, a ; x)$ by

$$
\sum_{n=0}^{\infty} \frac{t^{n}}{n !} \mathbf{C}_{n}(A ; \lambda, a ; x)=a^{t}\left(1-\frac{t}{\lambda x}\right)^{A}, x>0,\left|\frac{t}{\lambda x}\right|<1, \lambda x \neq 0, a>0, a \neq 1
$$

From (2.1), we obtain a series representation of Charlier matrix polynomials of degree $n$ as

$$
\mathbf{C}_{n}(A ; \lambda, a ; x)=n ! \sum_{k=0}^{n} \frac{(-1)^{k}}{k !(n-k) !} \Gamma(A+I) \Gamma^{-1}(A-(k-1) I)(\log (a))^{n-k}(\lambda x)^{-k} .
$$

Theorem 2.1. Charlier matrix polynomials is defined by

$$
\mathbf{C}_{n}(A ; \lambda, a ; x)=(\log (a))_{2}^{n} F_{0}\left(-n I,-A ;-;-\frac{1}{\lambda x \log (a)}\right), \lambda x \log (a) \neq 0 .
$$

Proof. Using the relations (1.2) and (1.4) in (2.2), we get

$$
\mathbf{C}_{n}(A ; \lambda, a ; x)=\sum_{k=0}^{n} \frac{1}{k !}(-n I)_{k}(-1)^{k}(-A)_{k}(\log (a))^{n-k}(\lambda x)^{-k}
$$

the representation (2.3) is obtained. 
Theorem 2.2. The generating matrix function for Charlier matrix polynomials is

$$
\sum_{n=0}^{\infty} \frac{t^{n}}{n !}(B){ }_{n} \mathbf{C}_{n}(A ; \lambda, a ; x)=(1-t \log (a))^{-B}{ }_{2} F_{0}\left(B,-A ;-; \frac{t}{\lambda x(1-t \log (a))}\right),|t \log (a)|<1
$$

where $B$ is a matrix in $\mathbb{C}^{N \times N}$.

Proof. By using the series representation of Charlier matrix polynomials given in (2.2) and (1.2), we get

$$
\begin{aligned}
\sum_{n=0}^{\infty} \frac{t^{n}}{n !}(B)_{n} \mathbf{C}_{n}(A ; \lambda, a ; x) & =\sum_{n=0}^{\infty} \sum_{k=0}^{n} \frac{(-1)^{k}}{k !(n-k) !}(B)_{n}(-1)^{k}(-A)_{k}(\log (a))^{n-k}(\lambda x)^{-k} t^{n} \\
& =\sum_{n=0}^{\infty} \sum_{k=0}^{\infty} \frac{1}{k ! n !}(B)_{k}(B+k I)_{n}(-A)_{k}(\log (a))^{n}(\lambda x)^{-k} t^{n+k} \\
& =\sum_{k=0}^{\infty} \frac{1}{k !}(B)_{k}(-A)_{k}(\log (a))^{n}(\lambda x)^{-k}(1-t \log (a))^{-(B+k I)} t^{k} \\
& =(1-t \log (a))^{-B} \sum_{k=0}^{\infty} \frac{1}{k !}(B)_{k}(-A)_{k}(\lambda x)^{-k}\left(\frac{t}{1-t \log (a)}\right)^{k}
\end{aligned}
$$

which gives the desired result. The matrix recurrence relations are also presented in the next theorem:

Theorem 2.3. For the Charlier matrix polynomials, the matrix recurrence relations

$$
\begin{aligned}
& x \frac{d}{d x} \mathbf{C}_{n}(A ; \lambda, a ; x)+n \mathbf{C}_{n}(A ; \lambda, a ; x)=n \log (a) \mathbf{C}_{n-1}(A ; \lambda, a ; x), n \geq 1, \\
& x \frac{d}{d x} \mathbf{C}_{n}(A ; \lambda, a ; x)+(A-\lambda x \log (a) I) \mathbf{C}_{n}(A ; \lambda, a ; x)=-\lambda x \mathbf{C}_{n+1}(A ; \lambda, a ; x)
\end{aligned}
$$

and

$$
\lambda x \mathbf{C}_{n+1}(A ; \lambda, a ; x)+n \log (a) \mathbf{C}_{n-1}(A ; \lambda, a ; x)+(A-(n+\lambda x \log (a)) I) \mathbf{C}_{n}(A ; \lambda, a ; x)=\boldsymbol{O}, n \geq 1,
$$

hold.

Proof. Differentiating (2.1) with respect to $x$, we can write

$$
\sum_{n=0}^{\infty} \frac{t^{n}}{n !} \frac{d}{d x} \mathbf{C}_{n}(A ; \lambda, a ; x)=\frac{t}{\lambda x^{2}} A a^{t}\left(1-\frac{t}{\lambda x}\right)^{A-I}
$$

Multiplying equation (2.8) by $\left(1-\frac{t}{\lambda x}\right)$ and using (2.1), we get

$$
\sum_{n=0}^{\infty} \frac{t^{n}}{n !} \frac{d}{d x} \mathbf{C}_{n}(A ; \lambda, a ; x)-\frac{1}{\lambda x} \sum_{n=0}^{\infty} \frac{t^{n+1}}{n !} \frac{d}{d x} \mathbf{C}_{n}(A ; \lambda, a ; x)=\frac{A}{\lambda x^{2}} \sum_{n=0}^{\infty} \frac{t^{n+1}}{n !} \mathbf{C}_{n}(A ; \lambda, a ; x) .
$$

Comparing the coefficient of $t^{n}$ yields the matrix differential recurrence relation

$$
\lambda x^{2} \frac{d}{d x} \mathbf{C}_{n}(A ; \lambda, a ; x)-n x \frac{d}{d x} \mathbf{C}_{n-1}(A ; \lambda, a ; x)=n A \mathbf{C}_{n-1}(A ; \lambda, a ; x)
$$


On the other hand, differentiating (2.1) with respect to $t$, we have

$$
\sum_{n=0}^{\infty} \frac{n t^{n-1}}{n !} \mathbf{C}_{n}(A ; \lambda, a ; x)=-\frac{A}{\lambda x} a^{t}\left(1-\frac{t}{\lambda x}\right)^{A-I}+\log (a) a^{t}\left(1-\frac{t}{\lambda x}\right)^{A} .
$$

Using (2.8) into (2.10) and subtracting

$$
\sum_{n=0}^{\infty} \frac{n t^{n}}{n !} \mathbf{C}_{n}(A ; \lambda, a ; x)=-x \sum_{n=0}^{\infty} \frac{t^{n}}{n !} \frac{d}{d x} \mathbf{C}_{n}(A ; \lambda, a ; x)+\log (a) \sum_{n=0}^{\infty} \frac{t^{n+1}}{n !} \mathbf{C}_{n}(A ; \lambda, a ; x) .
$$

Comparing the coefficient of $t^{n}$, we obtain (2.5).

Multiplying equation (2.5) by $\lambda x$, using (2.9) and subtracting $\frac{d}{d x} \mathbf{C}_{n}(A ; \lambda, a ; x)$, we have

$$
x \frac{d}{d x} \mathbf{C}_{n-1}(A ; \lambda, a ; x)+(A-\lambda x \log (a)) \mathbf{C}_{n-1}(A ; \lambda, a ; x)=-\lambda x A \mathbf{C}_{n}(A ; \lambda, a ; x) .
$$

Replacing $n$ by $n+1$ in (2.11), we obtain (2.6). From (2.5) and (2.6), we derive the desired pure matrix recurrence relation (2.7).

Theorem 2.4. The finite summation for Charlier matrix polynomials is

$$
\left(1-\frac{y}{x}\right)^{n} \mathbf{C}_{n}(A ; \lambda, a ; x-y)=\sum_{k=0}^{n} \frac{n !}{k !(n-k) !}\left(-\frac{y \log (a)}{x}\right)^{k} \mathbf{C}_{n-k}(A ; \lambda, a ; x),\left|\frac{y}{x}\right|<1, x \neq 0 .
$$

Proof. In (2.1), taking $u=\frac{t}{\lambda x}$, we can write the generating matrix relation

$$
\sum_{n=0}^{\infty} \frac{u^{n}}{n !}(\lambda x)^{n} \mathbf{C}_{n}(A ; \lambda, a ; x)=a^{\lambda x u}(1-u)^{A}, x>0,|u|<1
$$

Let us consider the following equation:

$$
\sum_{n=0}^{\infty} \frac{u^{n}}{n !}(\lambda x)^{n} \mathbf{C}_{n}(A ; \lambda, a ; x)=a^{\lambda y u} \sum_{n=0}^{\infty} \frac{u^{n}}{n !}(\lambda(x-y))^{n} \mathbf{C}_{n}(A ; \lambda, a ; x-y) .
$$

Multiplying the both sides of this equation by $a^{-\lambda y u}$, replacing $n$ by $n-k$ and using (2.13), we give

$$
\begin{aligned}
& \sum_{n=0}^{\infty} \frac{u^{n}}{n !}(\lambda(x-y))^{n} \mathbf{C}_{n}(A ; \lambda, a ; x-y)=a^{-\lambda y u} \sum_{n=0}^{\infty} \frac{u^{n}}{n !}(\lambda x)^{n} \mathbf{C}_{n}(A ; \lambda, a ; x) \\
& =\sum_{n=0}^{\infty} \sum_{k=0}^{\infty} \frac{u^{n+k}}{k ! n !}(-\lambda y \log (a))^{k}(\lambda x)^{n} \mathbf{C}_{n}(A ; \lambda, a ; x) \\
& =\sum_{n=0}^{\infty} \sum_{k=0}^{n} \frac{u^{n}}{k !(n-k) !}(-\lambda y \log (a))^{k}(\lambda x)^{n-k} \mathbf{C}_{n-k}(A ; \lambda, a ; x) .
\end{aligned}
$$

Comparing the coefficient of $t^{n}$, the relation is established.

Corollary 2.1. The Charlier matrix polynomials satisfy

$$
(1+t)^{n} \mathbf{C}_{n}(A ; \lambda, a ; x(1+t))=\sum_{k=0}^{n} \frac{n !}{k !(n-k) !}(t \log (a))^{k} \mathbf{C}_{n-k}(A ; \lambda, a ; x),|t|<1 .
$$

Proof. Taking $-\frac{y}{x} \longrightarrow t$ in equation (2.12), we have the desired result. 
Theorem 2.5. The generating matrix relation for Charlier matrix polynomials is

$$
\sum_{k=0}^{\infty} \frac{1}{k !} \mathbf{C}_{n+k}(A ; \lambda, a ; x) t^{k}=a^{t}\left(1-\frac{t}{\lambda x}\right)^{A} \mathbf{C}_{n}\left(A ; \lambda, a ; x\left(1-\frac{t}{\lambda x}\right)\right) ;\left|\frac{t}{\lambda x}\right|<1 .
$$

Proof. If we replace $t$ by $t+v$ in (2.1):

$$
a^{t+v}\left(1-\frac{t+v}{\lambda x}\right)^{A}=\sum_{n=0}^{\infty} \frac{(t+v)^{n}}{n !} \mathbf{C}_{n}(A ; \lambda, a ; x) .
$$

By expanding the binomial theorem $(t+v)^{n}$, replacing $n$ by $n+k$ and simplifying, we can write

$$
\begin{aligned}
& \sum_{n=0}^{\infty} \frac{(t+v)^{n}}{n !} \mathbf{C}_{n}(A ; \lambda, a ; x)=\sum_{n=0}^{\infty} \sum_{k=0}^{n} \frac{1}{k !(n-k) !} \mathbf{C}_{n+k}(A ; \lambda, a ; x) t^{k} v^{n} \\
& =\sum_{n=0}^{\infty} \sum_{k=0}^{\infty} \frac{1}{n ! k !} \mathbf{C}_{n+k}(A ; \lambda, a ; x) t^{k} v^{n} .
\end{aligned}
$$

If we associate the factors depending on $v$ in the left member of (2.16), we get

$$
a^{t+v}\left(1-\frac{t+v}{\lambda x}\right)^{A}=a^{t}\left(1-\frac{t}{\lambda x}\right)^{A} a^{v}\left(1-\frac{v}{\lambda x\left(1-\frac{t}{\lambda x}\right)}\right)^{A}=\sum_{n=0}^{\infty} \frac{(t+v)^{n}}{n !} \mathbf{C}_{n}(A ; \lambda, a ; x) .
$$

By means of (2.1), we can write

$$
a^{v}\left(1-\frac{v}{\lambda x\left(1-\frac{t}{\lambda x}\right)}\right)^{A}=\sum_{n=0}^{\infty} \frac{v^{n}}{n !} \mathbf{C}_{n}\left(A ; \lambda, a ; x\left(1-\frac{t}{\lambda x}\right)\right) .
$$

Therefore, the left member of (2.16) has the expansion in powers of $v$ :

$$
a^{t+v}\left(1-\frac{t+v}{\lambda x}\right)^{A}=a^{t}\left(1-\frac{t}{\lambda x}\right)^{A} \sum_{n=0}^{\infty} \frac{v^{n}}{n !} \mathbf{C}_{n}\left(A ; \lambda, a ; x\left(1-\frac{t}{\lambda x}\right)\right) .
$$

Hence

$$
\sum_{n=0}^{\infty} \sum_{k=0}^{\infty} \frac{1}{n ! k !} \mathbf{C}_{n+k}(A ; \lambda, a ; x) t^{k} v^{n}=a^{t}\left(1-\frac{t}{\lambda x}\right)^{A} \sum_{n=0}^{\infty} \frac{v^{n}}{n !} \mathbf{C}_{n}\left(A ; \lambda, a ; x\left(1-\frac{t}{\lambda x}\right)\right) .
$$

Comparing the coefficients of $v^{n}$ in the above equation, we get the assertion $((2.15)$ of Theorem 2.5 .

Theorem 2.6. The Charlier matrix polynomials is a solution of second order matrix differential equation

$$
\left[x^{2} D^{2} I+((1+n-\lambda x \log (a)) I+A) x D+n A\right] \mathbf{C}_{n}(A ; \lambda, a ; x)=\boldsymbol{O} .
$$

Proof. Starting from (2.5) and (2.9), we get

$$
(A-\lambda x \log (a) I) x D \mathbf{C}_{n}(A ; \lambda, a ; x)+n A \mathbf{C}_{n}(A ; \lambda, a ; x)=-n x \log (a) D \mathbf{C}_{n-1}(A ; \lambda, a ; x) .
$$

Differentiating (2.5) with respect to $x$ and multiplying by $x$, we get

$$
x^{2} D^{2} \mathbf{C}_{n}(A ; \lambda, a ; x)+(n+1) x D \mathbf{C}_{n}(A ; \lambda, a ; x)=n x \log (a) D \mathbf{C}_{n-1}(A ; \lambda, a ; x) .
$$

Adding the result to (2.18) and (2.19) gives the second order matrix differential equation for the Charlier matrix polynomials. 
Starting from (2.2), we get the another formula of matrix recurrence relation for the Charlier matrix polynomials

$$
\mathbf{C}_{n+1}(A ; \lambda, a ; x)=\log (a) \mathbf{C}_{n}(A ; \lambda, a ; x)-\frac{A}{\lambda x} \mathbf{C}_{n}(A-I ; \lambda, a ; x), \mathbf{C}_{0}(A ; \lambda, a ; x)=I,
$$

and if we take the backward shift operator $\mathbb{E} f(A)=f(A-I)$ as

$$
\begin{array}{r}
\mathbf{C}_{n}(A ; \lambda, a ; x)=\left(\log (a) I-\frac{1}{\lambda x} A \mathbb{E}\right) \mathbf{C}_{n-1}(A ; \lambda, a ; x) \\
=\left(I-\frac{1}{\lambda x \log (a)} A \mathbb{E}\right) \log (a) \mathbf{C}_{n-1}(A ; \lambda, a ; x)
\end{array}
$$

which gives (2.20) and we obtain the new explicit representation for Charlier matrix polynomials

$$
\mathbf{C}_{n}(A ; \lambda, a ; x)=\left(I-\frac{1}{\lambda x \log (a)} A \mathbb{E}\right)^{n}(\log (a))^{n} .
$$

Expanding the right hand side of the previous expression, using the binomial expansion, yields the new explicit representation for Charlier matrix polynomials

$$
\begin{aligned}
\mathbf{C}_{n}(A ; \lambda, a ; x) & =\sum_{k=0}^{n} \frac{(-1)^{k} n !}{k !(n-k) !}\left(\frac{A \mathbb{E}}{\lambda x \log (a)}\right)^{k}(\log (a))^{n} \\
& =\sum_{k=0}^{n} \frac{(-1)^{k} n !}{k !(n-k) !}\left(\frac{\mathbb{Q}^{(k)}}{(\lambda x \log (a))^{k}}\right)(\log (a))^{n}
\end{aligned}
$$

where $\mathbb{Q}^{(0)}=I, \mathbb{Q}^{(1)}=A \mathbb{E} 1=A, \mathbb{Q}^{(2)}=(A \mathbb{E})^{2} 1=(A \mathbb{E})(A \mathbb{E}) 1=(A \mathbb{E}) A=A(A-I)$ and in general $\mathbb{Q}^{(k)}=(A \mathbb{E})^{k} 1=A(A-I) \ldots(A-(k-1) I)$.

Thus, we list the first elements of the Chartier matrix polynomials

$$
\begin{aligned}
\mathbf{C}_{0}(A ; \lambda, a ; x) & =I=\mathbb{Q}^{(0)}, \\
\mathbf{C}_{1}(A ; \lambda, a ; x) & =\frac{A}{\lambda x}-\log (a) I=\left(\frac{A}{\lambda x \log (a)}-I\right) \log (a)=\left(\frac{\mathbb{Q}^{(1)}}{\lambda x \log (a)}-\mathbb{Q}^{(0)}\right) \log (a) \\
\mathbf{C}_{2}(A ; \lambda, a ; x) & =\left(\frac{A(A-I)}{(\lambda x \log (a))^{2}}-2 \frac{A}{\lambda x \log (a)}+I\right)(\log (a))^{2}=\left(\frac{\mathbb{Q}^{(2)}}{(\lambda x \log (a))^{2}}-2 \frac{\mathbb{Q}^{(1)}}{\lambda x \log (a)}+\mathbb{Q}^{(0)}\right)(\log (a))^{2}, \\
\mathbf{C}_{3}(A ; \lambda, a ; x) & =\left(\frac{A(A-I)(A-2 I)}{(\lambda x \log (a))^{3}}-3 \frac{A(A-I)}{(\lambda x \log (a))^{2}}+3 \frac{A}{\lambda x \log (a)}-I\right)(\log (a))^{3} \\
& =\left(\frac{\mathbb{Q}^{(3)}}{(\lambda x \log (a))^{3}}-3 \frac{\mathbb{Q}^{(2)}}{(\lambda x \log (a))^{2}}+3 \frac{\mathbb{Q}^{(1)}}{\lambda x \log (a)}-\mathbb{Q}^{(0)}\right)(\log (a))^{3} .
\end{aligned}
$$

Summary of these results are given in the next theorem.

Theorem 2.7. For a matrix $A$ in $\mathbb{C}^{N \times N}$, then Chartier matrix polynomials satisfy:

- For $n \geq 1$

$$
\mathbf{C}_{n+1}(A ; \lambda, a ; x)=\log (a) \mathbf{C}_{n}(A ; \lambda, a ; x)-\frac{A}{\lambda x} \mathbf{C}_{n}(A-I ; \lambda, a ; x), \mathbf{C}_{0}(A ; \lambda, a ; x)=I .
$$

- In (2.21) and (2.22) are defined the matrix versions of new explicit representations for Charlier matrix polynomials. 


\section{$3 \quad$ Linear differential operators}

In accordance with the discussion in the preceding section, the partial differential matrix equation are constructed from (2.17) by replacing $n$ by $y \frac{\partial}{\partial y}, D$ by $\frac{\partial}{\partial x}$ and $\mathbf{C}_{n}(A ; \lambda, a ; x)$ by $\mathbf{C}_{n}(A ; \lambda, a ; x, y)=$ $y^{n} \mathbf{C}_{n}(A ; \lambda, a ; x)$ :

$$
\left[x^{2} \frac{\partial^{2}}{\partial x^{2}} I+\left(\left(1+y \frac{\partial}{\partial y}-\lambda x \log (a)\right) I+A\right) x \frac{\partial}{\partial x}+A y \frac{\partial}{\partial y}\right] \mathbf{C}_{n}(A ; \lambda, a ; x, y)=\mathbf{0} .
$$

We rewrite (3.1) in the form

$$
\left[x^{2} \frac{\partial^{2}}{\partial x^{2}} I+((1-\lambda x \log (a)) I+A) x \frac{\partial}{\partial x}+x y \frac{\partial}{\partial y \partial x} I+A y \frac{\partial}{\partial y}\right] \mathbf{C}_{n}(A ; \lambda, a ; x, y)=\mathbf{0} .
$$

Since

$$
\mathbb{L}\left(x, \frac{\partial}{\partial x}, y \frac{\partial}{\partial y}\right)\left[\mathbf{C}_{n}(A ; \lambda, a ; x) y^{n}\right]=y^{n} \mathbb{L}\left(x, \frac{d}{d x}, n\right)\left[\mathbf{C}_{n}(A ; \lambda, a ; x)\right],
$$

for $y \neq 0$, we get

$$
\mathbb{L}\left(x, \frac{\partial}{\partial x}, y \frac{\partial}{\partial y}\right)\left[\mathbf{C}_{n}(A ; \lambda, a ; x) y^{n}\right]=\mathbf{0},
$$

if and only if

$$
\mathbb{L}\left(x, \frac{d}{d x}, n\right)\left[\mathbf{C}_{n}(A ; \lambda, a ; x)\right]=\mathbf{0} .
$$

Again using the technique described in detail in section 2, we determine with the aid of (2.5) and (2.6) the first order linear differential operators for Charlier matrix polynomials $\mathbb{I}, \mathbb{A}, \mathbb{B}$ and $\mathbb{C}$ such that

$$
\begin{aligned}
& \mathbb{A}\left[\mathbf{C}_{n}(A ; \lambda, a ; x) y^{n}\right]=n \mathbf{C}_{n}(A ; \lambda, a ; x) y^{n}, \\
& \mathbb{B}\left[\mathbf{C}_{n}(A ; \lambda, a ; x) y^{n}\right]=n \log (a) \mathbf{C}_{n-1}(A ; \lambda, a ; x) y^{n-1}
\end{aligned}
$$

and

$$
\mathbb{C}\left[\mathbf{C}_{n}(A ; \lambda, a ; x) y^{n}\right]=-\lambda \mathbf{C}_{n+1}(A ; \lambda, a ; x) y^{n+1},
$$

where

$$
\begin{aligned}
& \mathbb{A}=y \frac{\partial}{\partial y} I, \\
& \mathbb{B}=\frac{x}{y} \frac{\partial}{\partial x} I+\frac{\partial}{\partial y} I
\end{aligned}
$$

and

$$
\mathbb{C}=y \frac{\partial}{\partial x} I+y\left(\frac{1}{x} A-\lambda \log (a) I\right) .
$$

Therefore, the operators $\mathbb{I}, \mathbb{A}, \mathbb{B}$ and $\mathbb{C}$ generate a Lie group. From the above commutator relations, we can state the next theorem. 
Theorem 3.1. The set of linear combinations of the partial differential operators $\mathbb{I}, \mathbb{A}, \mathbb{B}$ and $\mathbb{C}$ forms a Lie algebra.
(i) $[\mathbb{A}, \mathbb{B}]=-\mathbb{B}$,
(ii) $[\mathbb{A}, \mathbb{C}]=\mathbb{C}$,
(iii) $\quad[\mathbb{B}, \mathbb{C}]=-\lambda \log (a) \mathbb{I}$,

where $\mathbb{I}$ stands for the identity operator.

Proof. We find that

$$
\mathbb{A B} \mathbf{C}_{n}(A ; \lambda, a ; x, y)=y \frac{\partial}{\partial y}\left[\frac{x}{y} \frac{\partial}{\partial x} I+\frac{\partial}{\partial y} I\right] \mathbf{C}_{n}(A ; \lambda, a ; x, y) .
$$

Hence, on simplification, we have

$$
\mathbb{A B} \mathbf{C}_{n}(A ; \lambda, a ; x, y)=x \frac{\partial^{2}}{\partial y \partial x} \mathbf{C}_{n}(A ; \lambda, a ; x, y)-\frac{x}{y} \frac{\partial}{\partial x} \mathbf{C}_{n}(A ; \lambda, a ; x, y)+y \frac{\partial^{2}}{\partial y^{2}} \mathbf{C}_{n}(A ; \lambda, a ; x, y) .
$$

On the other hand, we have

$$
\mathbb{B} \mathbb{A} \mathbf{C}_{n}(A ; \lambda, a ; x, y)=\left(\frac{x}{y} \frac{\partial}{\partial x} I+\frac{\partial}{\partial y} I\right) y \frac{\partial}{\partial y} \mathbf{C}_{n}(A ; \lambda, a ; x, y) .
$$

This is to be simplified as

$$
\mathbb{B} A \mathbf{C}_{n}(A ; \lambda, a ; x, y)=x \frac{\partial^{2}}{\partial x \partial y} \mathbf{C}_{n}(A ; \lambda, a ; x, y)+\frac{\partial}{\partial y} \mathbf{C}_{n}(A ; \lambda, a ; x, y)+y \frac{\partial^{2}}{\partial y^{2}} \mathbf{C}_{n}(A ; \lambda, a ; x, y) .
$$

Then for $\frac{\partial^{2}}{\partial x \partial y}=\frac{\partial^{2}}{\partial y \partial x}$, subtracting (3.11) from (3.10), we get

$$
\begin{aligned}
& {[\mathbb{A}, \mathbb{B}] \mathbf{C}_{n}(A ; \lambda, a ; x, y)=(\mathbb{A B}-\mathbb{B} \mathbb{A}) \mathbf{C}_{n}(A ; \lambda, a ; x, y)} \\
& =-\frac{x}{y} \frac{\partial}{\partial x} \mathbf{C}_{n}(A ; \lambda, a ; x, y)-\frac{\partial}{\partial y} \mathbf{C}_{n}(A ; \lambda, a ; x, y)=-\mathbb{B} \mathbf{C}_{n}(A ; \lambda, a ; x, y) .
\end{aligned}
$$

Thus, we have the required result $[\mathbb{A}, \mathbb{B}]=-\mathbb{B}$. Similarly, we can proceed to calculate each of the following results $[\mathbb{A}, \mathbb{C}]$ and $[\mathbb{B}, \mathbb{C}]$, which completes the proof theorem.

Now, we know that the operator $\frac{1}{x} \mathbb{L}$ given by

$$
\begin{aligned}
\frac{1}{x} \mathbb{L} \mathbf{C}_{n}(A ; \lambda, a ; x, y)= & x \frac{\partial^{2}}{\partial x^{2}} \mathbf{C}_{n}(A ; \lambda, a ; x, y)+((1-\lambda x \log (a)) I+A) \frac{\partial}{\partial x} \mathbf{C}_{n}(A ; \lambda, a ; x, y) \\
& +y \frac{\partial}{\partial y \partial x} \mathbf{C}_{n}(A ; \lambda, a ; x, y)+\frac{A y}{x} \frac{\partial}{\partial y} \mathbf{C}_{n}(A ; \lambda, a ; x, y) .
\end{aligned}
$$

Also, in making the calculation of $[\mathbb{B}, \mathbb{C}]$, we found that

$$
\begin{aligned}
& \mathbb{C B} \mathbf{C}_{n}(A ; \lambda, a ; x, y)=x \frac{\partial^{2}}{\partial x^{2}} \mathbf{C}_{n}(A ; \lambda, a ; x, y)+(1+A-\lambda x \log (a)) \frac{\partial}{\partial x} \mathbf{C}_{n}(A ; \lambda, a ; x, y) \\
& +y \frac{\partial^{2}}{\partial x \partial y} \mathbf{C}_{n}(A ; \lambda, a ; x, y)+\left(\frac{A}{x}-\lambda \log (a)\right) y \frac{\partial}{\partial y} \mathbf{C}_{n}(A ; \lambda, a ; x, y) .
\end{aligned}
$$

Hence, we get

$$
\frac{1}{x} \mathbb{L} \mathbf{C}_{n}(A ; \lambda, a ; x, y)-\mathbb{C B} \mathbf{C}_{n}(A ; \lambda, a ; x, y)=\lambda \log (a) y \frac{\partial}{\partial y} \mathbf{C}_{n}(A ; \lambda, a ; x, y) .
$$


Therefore, it can be expressed as:

$$
\frac{1}{x} \mathbb{L} \mathbf{C}_{n}(A ; \lambda, a ; x, y)=[\mathbb{C B}+\lambda \log (a) \mathbb{A}] \mathbf{C}_{n}(A ; \lambda, a ; x, y),
$$

or equivalently,

$$
\frac{1}{x} \mathbb{L}=\mathbb{C B}+\lambda \log (a) \mathbb{A} ; x \neq 0 .
$$

Now, we show that

$$
\begin{aligned}
{\left[\frac{1}{x} \mathbb{L}, \mathbb{C}\right] \mathbf{C}_{n}(A ; \lambda, a ; x, y) } & =\left(\frac{1}{x} \mathbb{L} \mathbb{C}-\mathbb{C} \frac{1}{x} \mathbb{L}\right) \mathbf{C}_{n}(A ; \lambda, a ; x, y) \\
& =((\mathbb{C B}+\lambda \log (a) \mathbb{A}) \mathbb{C}-\mathbb{C}(\mathbb{C B}+\lambda \log (a) \mathbb{A})) \mathbf{C}_{n}(A ; \lambda, a ; x, y) \\
& =(\mathbb{C B} \mathbb{C}+\lambda \log (a) \mathbb{A}-\mathbb{C} \mathbb{C B}-\mathbb{C} \lambda \log (a) \mathbb{A}) \mathbf{C}_{n}(A ; \lambda, a ; x, y)
\end{aligned}
$$

With the help of (3.9), we simplify $\mathbb{A} \mathbb{C}-\mathbb{C A}$ as follows:

$$
\mathbb{A} \mathbb{C}-\mathbb{C} A=\mathbb{C} .
$$

Hence, with the further aid of the commutator relations from (3.9), we get

$$
\mathbb{C}(\mathbb{B C}-\mathbb{C B})=\mathbb{C}\left(-\lambda \log (a) \mathbf{C}_{n}(A ; \lambda, a ; x, y)\right)=-\lambda \log (a) \mathbb{C}
$$

From (3.13), we obtain

$$
\left[\frac{1}{x} \mathbb{L}, \mathbb{C}\right]=\mathbf{0}
$$

Thus, we establish that the operator $\frac{1}{x} \mathbb{L}$ commutes with $\mathbb{C}$. In the same way, we can calculate each of the following results $\left[\frac{1}{x} \mathbb{L}, \mathbb{A}\right]=\mathbf{0}$ and $\left[\frac{1}{x} \mathbb{L}, \mathbb{B}\right]=\mathbf{0}$. These results are summarized in the following theorem. We summarize these results in the following theorem.

Theorem 3.2. Linear differential operators $\mathbb{A}, \mathbb{B}$ and $\mathbb{C}$ defined in (3.5), (3.6) and (3.7) commute with the operator $\frac{1}{x} \mathbb{L}$ as follows

$$
\text { (i) } \quad\left[\frac{1}{x} \mathbb{L}, \mathbb{A}\right]=\mathbf{0}, \quad(\text { ii }) \quad\left[\frac{1}{x} \mathbb{L}, \mathbb{B}\right]=\mathbf{0}, \quad \text { (iii) } \quad\left[\frac{1}{x} \mathbb{L}, \mathbb{C}\right]=\mathbf{0},
$$

where the operator $\mathbb{L}$ is as defined in (3.2).

We express the extended form of the group generated by the operators $\mathbb{I}, \mathbb{A}, \mathbb{B}$ and $\mathbb{C}$ as follows:

$$
\begin{aligned}
e^{a \mathbb{A}} f(x, y, A) & =f\left(x, y e^{a}, A\right), \\
e^{b \mathbb{B}} f(x, y, A) & =f\left(x\left(1+\frac{b}{y}\right), b+y, A\right), y \neq 0,
\end{aligned}
$$

and

$$
e^{c \mathbb{C}} f(x, y, A)=e^{-\lambda c y \log (a)}\left(1+\frac{c y}{x}\right)^{A} f(x+c y, y, A) ; x \neq 0,\left|\frac{c y}{x}\right|<1
$$


where $f(x, y, A)$ is an arbitrary matrix function and $a, b$ and $c$ are arbitrary constants.

From the above relations, we get

$$
e^{c \mathbb{C}} e^{b \mathbb{B}} e^{a \mathbb{A}} f(x, y, A)=e^{-\lambda c y \log (a)}\left(1+\frac{c y}{x}\right)^{A} f\left((x+c y)\left(1+\frac{b}{y}\right),(b+y) e^{a}, A\right) ; x, y \neq 0,\left|\frac{c y}{x}\right|<1
$$

\subsection{Generating matrix functions derived from the operators}

Here, we have determined the new generating matrix relations between Charlier matrix polynomials. For arbitrary constants $b$ and $c$ the operator $e^{c \mathbb{C}} e^{b \mathbb{B}} \frac{1}{x} \mathbb{L}\left[\mathbf{C}_{n}(A ; \lambda, a ; x) y^{n}\right]$ will transform solutions of $\mathbb{L}$ into solutions of $\mathbb{L}$; in other words, we have

$$
e^{c \mathbb{C}} e^{b \mathbb{B}} \frac{1}{x} \mathbb{L} \mathbf{C}_{n}(A ; \lambda, a ; x, y)=\frac{1}{x} \mathbb{L} e^{c \mathbb{C}} e^{b \mathbb{B}} \mathbf{C}_{n}(A ; \lambda, a ; x, y)
$$

if and only if $\mathbb{L} u=\mathbf{0}$

Now it follows from $(3.3)$ that $\mathbf{C}_{n}(A ; \lambda, a ; x, y)$ is a solution of the system

$$
\mathbb{L} \mathbf{C}_{n}(A ; \lambda, a ; x, y)=\mathbf{0} \quad \text { and } \quad(\mathbb{A}-n \mathbb{I}) \mathbf{C}_{n}(A ; \lambda, a ; x, y)=\mathbf{0} .
$$

for the matrix function $\mathbf{C}_{n}(A ; \lambda, a ; x, y)=y^{n} \mathbf{C}_{n}(A ; \lambda, a ; x)$. Since $\mathbb{B}$ and $\mathbb{C}$ commute with $\frac{1}{x} \mathbb{L}$, we have

$$
e^{c \mathbb{C}} e^{b \mathbb{B}} \frac{1}{x} \mathbb{L}\left[\mathbf{C}_{n}(A ; \lambda, a ; x) y^{n}\right]=\frac{1}{x} \mathbb{L} e^{c \mathbb{C}} e^{b \mathbb{B}} \mathbb{L}\left[\mathbf{C}_{n}(A ; \lambda, a ; x) y^{n}\right]
$$

Before discussing particular cases (3.18), it is of interest to mention that the operators $\mathbb{B}$ and $\mathbb{C}$ being commutative. The above generating matrix relations which yield a good number of a particular generating relations by attributing different values to $a=0$ appears to be new. Therefore, we will consider the following three cases of the transformed function $e^{c \mathbb{C}} e^{b \mathbb{B}} \frac{1}{x} \mathbb{L}\left[\mathbf{C}_{n}(A ; \lambda, a ; x) y^{n}\right]$ :

Case 1. $b=1, c=0$,

Case 2. $b=0, c=1$.

Case 3. $b c \neq 0$

Case 1. From (3.16), we know that for an arbitrary matrix function

$$
e^{\mathbb{B}} f(x, y, A)=f\left(x\left(1+\frac{1}{y}\right), 1+y, A\right),
$$

then it follows that

$$
e^{\mathbb{B}}\left[\mathbf{C}_{n}(A ; \lambda, a ; x) y^{n}\right]=(1+y)^{n} \mathbf{C}_{n}\left(A ; \lambda, a ; x\left(1+\frac{1}{y}\right)\right) .
$$

Thus, we have obtained the generating matrix function which has the expansion:

$$
(1+y)^{n} \mathbf{C}_{n}\left(A ; \lambda, a ; x\left(1+\frac{1}{y}\right)\right)=\sum_{k=0}^{n} \frac{n !}{(n-k) ! k !}(\log (a))^{k} y^{n-k} \mathbf{C}_{n-k}(A ; \lambda, a ; x),
$$

If we divide by $y^{n}$

$$
\left(1+\frac{1}{y}\right)^{n} \mathbf{C}_{n}\left(A ; \lambda, a ; x\left(1+\frac{1}{y}\right)\right)=\sum_{k=0}^{n} \frac{n !}{(n-k) ! k !}(\log (a))^{k} y^{-k} \mathbf{C}_{n-k}(A ; \lambda, a ; x)
$$

Replacing $y^{-1}$ by $t$, we get the generating matrix relation

$$
(1+t)^{n} \mathbf{C}_{n}(A ; \lambda, a ; x(1+t))=\sum_{k=0}^{n} \frac{n !}{(n-k) ! k !}(\log (a))^{k} t^{k} \mathbf{C}_{n-k}(A ; \lambda, a ; x)
$$


Case 2. From (2.17) for an arbitrary matrix function, we know that

$$
e^{c \mathbb{C}} f(x, y, A)=e^{-\lambda c y \log (a)}\left(1+\frac{c y}{x}\right)^{A} f(x+c y, y, A) .
$$

Therefore, for $c=1$, we have

$$
\begin{aligned}
e^{\mathbb{C}}\left[\mathbf{C}_{n}(A ; \lambda, a ; x) y^{n}\right] & =e^{-\lambda y \log (a)}\left(1+\frac{y}{x}\right)^{A} \mathbf{C}_{n}(A ; \lambda, a ; x+y, y) \\
& =y^{n} e^{-\lambda y \log (a)}\left(1+\frac{y}{x}\right)^{A} \mathbf{C}_{n}(A ; \lambda, a ; x+y)
\end{aligned}
$$

If we equate the two expansions of this matrix function, we get

$$
y^{n} e^{-\lambda y \log (a)}\left(1+\frac{y}{x}\right)^{A} \mathbf{C}_{n}(A ; \lambda, a ; x+y)=\sum_{k=0}^{\infty} \frac{(-\lambda)^{k}}{k !} y^{n+k} \mathbf{C}_{n+k}(A ; \lambda, a ; x) .
$$

If we divide both members of this equation by $y^{n}$ and simplify, we obtain the generating matrix relation:

$$
e^{-\lambda y \log (a)}\left(1+\frac{y}{x}\right)^{A} \mathbf{C}_{n}(A ; \lambda, a ; x+y)=\sum_{k=0}^{\infty} \frac{(-\lambda)^{k}}{k !} y^{k} \mathbf{C}_{n+k}(A ; \lambda, a ; x)
$$

If $n=0$, this relation becomes the familiar generating matrix functions

$$
e^{-\lambda y \log (a)}\left(1+\frac{y}{x}\right)^{A} \mathbf{C}_{0}(A ; \lambda, a ; x+y)=\sum_{k=0}^{\infty} \frac{(-\lambda)^{k}}{k !} y^{k} \mathbf{C}_{k}(A ; \lambda, a ; x)
$$

Case 3. For $b c \neq 0$. In order to simplify results we choose $c=1$ and $b=-\frac{1}{\alpha}$. Then for all finite values of $\alpha$, we have $b c \neq 0$. For any arbitrary matrix function, then, we have

$$
\begin{array}{r}
e^{\mathbb{C}} e^{-\frac{1}{\alpha} \mathbb{B}} f(x, y, A)=e^{\mathbb{C}} f\left(x\left(1-\frac{1}{\alpha y}\right), y-\frac{1}{\alpha}, A\right) \\
=e^{-\lambda y \log (a)}\left(1+\frac{y}{x}\right)^{A} f\left((x+y)\left(1-\frac{1}{\alpha y}\right), y-\frac{1}{\alpha}, A\right) .
\end{array}
$$

Hence, we get

$$
e^{\mathbb{C}} e^{-\frac{1}{\alpha} \mathbb{B}}\left[\mathbf{C}_{n}(A ; \lambda, a ; x) y^{n}\right]=\left(y-\frac{1}{\alpha}\right)^{n} e^{-\lambda y \log (a)}\left(1+\frac{y}{x}\right)^{A} \mathbf{C}_{n}\left(A ; \lambda, a ;(x+y)\left(1-\frac{1}{\alpha y}\right)\right) .
$$

Separately, we consider the left hand side of (3.24) and we write exponential operators in a series 
form so that we have the following relation

$$
\begin{aligned}
e^{\mathbb{C}} e^{-\frac{1}{\alpha} \mathbb{B}}\left[\mathbf{C}_{n}(A ; \lambda, a ; x) y^{n}\right]=\sum_{r, k=0}^{\infty} \frac{1}{r ! k !}\left(-\frac{1}{\alpha}\right)^{k} \mathbb{C}^{r} \mathbb{B}^{k}\left[\mathbf{C}_{n}(A ; \lambda, a ; x) y^{n}\right] \\
=\sum_{r, k=0}^{\infty} \frac{1}{r ! k !}\left(-\frac{1}{\alpha}\right)^{k}(-\lambda)^{r} \mathbb{B}^{k}\left[\mathbf{C}_{n+r}(A ; \lambda, a ; x) y^{n+r}\right] \\
=\sum_{k=0}^{\infty} \sum_{r=0}^{n} \frac{1}{r ! k !}\left(-\frac{1}{\alpha}\right)^{k}(-\lambda)^{r} \mathbb{B}^{k}\left[\mathbf{C}_{n}(A ; \lambda, a ; x) y^{n}\right] \\
=\sum_{k=0}^{\infty} \sum_{r=0}^{n} \frac{1}{r ! k !}\left(-\frac{1}{\alpha}\right)^{k}(-\lambda)^{r}[n \log (a)(n-1) \log (a) \ldots(n-k+1) \log (a)] \mathbb{B}^{k-k}\left[\mathbf{C}_{n+k}(A ; \lambda, a ; x) y^{n+k}\right] \\
=\sum_{k=0}^{n} \sum_{r=0}^{n} \frac{n !}{(n-k) ! r ! k !}\left(-\frac{1}{\alpha}\right)^{k}(\log (a))^{k}(-\lambda)^{r} \mathbb{B}^{0}\left[\mathbf{C}_{n+k}(A ; \lambda, a ; x) y^{n+k}\right] \\
=\sum_{k=0}^{n} \sum_{r=0}^{n} \frac{n !}{(n-k) ! r ! k !}\left(-\frac{1}{\alpha}\right)^{k}(\log (a))^{k}(-\lambda)^{r} \mathbf{C}_{n+k}(A ; \lambda, a ; x) y^{n+k} \\
=\sum_{k=0}^{n} \sum_{r=0}^{n} \frac{(-1)^{k}}{r ! k !}(-n)_{k}\left(-\frac{1}{\alpha}\right)^{k}(\log (a))^{k}(-\lambda)^{r} \mathbf{C}_{n+k}(A ; \lambda, a ; x) y^{n+k} .
\end{aligned}
$$

Using (3.24) and (3.25), we get

$$
\begin{aligned}
& \left(y-\frac{1}{\alpha}\right)^{n} e^{-\lambda y \log (a)}\left(1+\frac{y}{x}\right)^{A} \mathbf{C}_{n}\left(A ; \lambda, a ;(x+y)\left(1-\frac{1}{\alpha y}\right)\right) \\
& =\sum_{k=0}^{n} \sum_{r=0}^{n} \frac{(-1)^{k}}{r ! k !}(-n)_{k}\left(-\frac{1}{\alpha}\right)^{k}(\log (a))^{k}(-\lambda)^{r} \mathbf{C}_{n+k}(A ; \lambda, a ; x) y^{n+k}
\end{aligned}
$$

Finally, we replace $y$ by $-t$ in above equation and simplifying, we obtained the generating matrix function between Charlier matrix polynomials, which we believe to be new generating function

$$
\begin{aligned}
& \left(t+\frac{1}{\alpha}\right)^{n} e^{\lambda t \log (a)}\left(1-\frac{t}{x}\right)^{A} \mathbf{C}_{n}\left(A ; \lambda, a ;(x-t)\left(1+\frac{1}{\alpha t}\right)\right) \\
& =\sum_{k=0}^{n} \sum_{r=0}^{n} \frac{(-1)^{r+k}(-n)_{k} \lambda^{r}(\log (a))^{k}}{r ! k ! \alpha^{k}} \mathbf{C}_{n+k}(A ; \lambda, a ; x) t^{n+k} .
\end{aligned}
$$

Certain generating matrix relations established in this discussion prove the usefulness of operators techniques in obtaining the results for the newly considered Charlier matrix polynomials from the corresponding ones holding the unknown and known Charlier matrix polynomials.

\section{Conclusion}

A novel approach has been adopted in this work for developing some important properties of Charlier matrix polynomials viz recurrence matrix relations, differential recurrence matrix relations and matrix differential matrix equation. Other important properties of the Charlier matrix polynomials will be obtained for further research work. Since the Charlier matrix polynomials are significant from the view point of applications. The Lie algebra method developed in this work can be used in the investigation of some other Charlier matrix polynomials which play a vital role in Mathematical Physics. 


\section{References}

[1] R. Agarwal and S. Jain, Certain properties of some special matrix functions via Lie Algebra, International Bulletin of Mathematical Research, Vol. 2, No. 1 (2015), pp. 9-15.

[2] C. V. L. Charlier, (1905-1906) Uber die Darstellung willkurlicher Funktionen, Ark. Mat. Astr. och Fysic, Vol. $\mathbf{2}, 20$.

[3] L. Jódar and J. C. Cortés, Some properties of gamma and beta matrix functions, Appl. Math. Lett., Vol. 11 (1998), pp. 89-93.

[4] L. Jódar and J. C. Cortés, On the hypergeometric matrix function, J. Comput. Appl. Math., Vol. 99 (1998), pp. 205-217.

[5] S. Khan and N. A. M. Hassan, 2-Variable Laguerre matrix polynomials and Lie-algebraic techniques, J. Phys. A., Vol. 43, No. 23 (2010), 235204 (21pp).

[6] S. Khan and N. Raza, 2-Variable generalized Hermite matrix polynomials and Lie algebra representation, Rep. Math. Phys., Vol. 66, No. 2 (2010), pp. 159-174.

[7] S. Khan and N. Raza, Hermite-Laguerre matrix polynomials and generating relations, Rep. Math. Phys., fVol. 73, No. 2 (2014), pp. 137-164.

[8] H. L. Manocha, Lie theoretic generating functions, Publ. Inst. Math. (Beograd) (N. S.), Vol. 20, No. 34 (1976), pp. $179-184$.

[9] H. L. Manocha and S. Jain, Special linear group and generating functions, Comment. Math. Univ. Str. Pauli., XXVI No. 1,(1977), pp. 105-113.

[10] E. B. McBride, Obtaining Generating Functions, Springer Verlag, New York, Heidelberg and Berlin, 1971.

[11] W. Miller, On Lie algebras and some special functions of mathematical physics, Amer. Math. Soc. Memo., No. 50, Amer. Math. Soc., Providence, R.I., 1964.

[12] W. Miller, Lie Theory and Special Functions, New York, Academic Press Inc., 1968

[13] S. Roman, The Poisson-Charlier Polynomials. 4.3.3 in The Umbral Calculus, New York: Academic Press, pp. 119-122, 1984.

[14] M. J. S. Shahwan and M. A. Pathan, Origin of certain generating relations of Hermite matrix functions from the view point of Lie Algebra, Integral Transforms Spec. Funct., Vol. 17, No. 10 (2006), pp. 743-747.

[15] M.J.S. Shahwan and M.A. Pathan, Generating relations of Hermite matrix polynomials by Lie Algebraic method, Ital. J. Pure Appl. Math., Vol. 25 (2009), pp. 187-192.

[16] A. Shehata, Some relations on Laguerre matrix polynomials, Malays. J. Math. Sci., Vol. 9, No. 3 (2015), pp. 443-462.

[17] A. Shehata, On modified Laguerre matrix polynomials, Journal of Natural Sciences and Mathematics, Vol. 8, No. 2 (2015), pp. 153-166.

[18] A. Shehata, Certain generating relations of generalized Bessel matrix polynomials from the view point of Lie algebra method, Bulletin of the Iranian Mathematical Society, Vol. 44, No. 4 (2018), pp. 1025-1043.

[19] A. Shehata, Certain generating relations of Konhauser matrix polynomials from the view point of Lie algebra method, Politehn. Univ. Bucharest Sci. Bull. Ser. A Appl. Math. Phys., Vol. 79, No. 4 (2017), pp. 123-136.

[20] A. Shehata, Certain properties of generalized Hermite-type matrix polynomials using Weisner's group theoretic techniques, Bulletin of the Brazilian Mathematical Society, New Series, Vol. 50 (2019), pp. 419-434.

[21] L. Weisner, Generating functions for Hermite functions, Canad. J. Math., Vol. 11 (1959), pp. 141-147.

[22] L. Weisner, Generating functions for Bessel functions, Canad. J. Math., Vol. 11 (1959), PP. 148-155.

[23] L. Weisner, Croup-theoretic origin of certain generating functions, Pacific J. Math., Vol. 5 (1955), pp. 10331039.

[24] L. Weisner, Lie theory and the hypergeometric functions, J. Math. Mech., Vol. 17 (1968), pp. 1143-1174.

[25] L. Weisner, Lie theory and some special solutions of the hypergeometric equation, SIAM J. Math. Anal., Vol. 1 (1970), pp. 405-425.

[26] L. Weisner, On Lie algebras of difference operators and the special functions of mathematical physics, SIAM J. Math. Anal., Vol. 2 (1971), pp. 307-327. 